\section{Viscosity of Air and the Electronic Charge}

As one of the possible explanations for the discrepancy between the "oil drop value" and the "x-ray value" of the electronic charge it has been suggested ${ }^{1}$ that Millikan has used too low a value for the coefficient of viscosity of air $\eta$, which enters as a factor of the power $3 / 2$ in calculating $e$ by the oil drop method. In order to examine this hypothesis, the author has undertaken a new determination of $\eta$, using the rotating cylinder method. As a preliminary result was published $^{2}$ the value

$$
\begin{aligned}
& \eta_{20}{ }^{\circ}=(18200 \pm 30) \cdot 10^{-8} \quad \text { corresponding to } \\
& \eta_{23^{\circ}}=(18348 \pm 30) \cdot 10^{-8} .
\end{aligned}
$$

This is 0.67 percent higher than the value used by Millikan in $1916\left(18226 \cdot 10^{-8}\right)$, and would give a value of $e 1.0$ percent higher than Millikan's.

Since this result was published, the apparatus has been improved so as to insure greater constancy of the temperature and of the speed of rotation of the outer cylinder. The mean of 54 determinations, carried out with the improved apparatus, is

$$
\eta_{20^{\circ}}=(18201 \pm 27) \cdot 10^{-8} \text { or } \eta_{23}{ }^{\circ}=(18349 \pm 27) \cdot 10^{-8}
$$

in good agreement with the previous value, and with somewhat greater accuracy.
If we use Millikan's data with this value of $\eta$ we would find for $e$

$$
e=\left(\frac{18349}{18226}\right)^{\frac{3}{2}} 4.770 \cdot 10^{-10}=(4.818 \pm 0.011) \cdot 10^{-10} \text { e.s.u. }
$$

the uncertainty stated being due only to the viscosity.

It should be emphasized, that the error in $\eta$ has been calculated so as to give a reasonable limit of error rather than a probable error, as some systematic errors have also been considered. (The probable accidental error, computed by the usual least square method from the individual deviations would be only $1.5 \cdot 10^{-8}$ or 0.008 percent.)

From this result the conclusion can be drawn, that the lower value of $e$ obtained by the oil drop method of Millikan can very, well be explained by the error in the $\eta$-value used. New measurements by Bäcklin and Flemberg ${ }^{3}$ seem to indicate that other errors may also exist.

A detailed report has been sent for publication elsewhere.

Gunnar Kellström

Physics Laboratory, University of Uppsala, Sweden, June 20, 1936.

1 K. Shiba, Sci. Pap. Inst. phys. chem. Res. Tokyo 19, 97 (1932), 2 G. Kellström, Nature 136, 682 (1935). 3 E. Bäcklin and H. Flemberg, Nature 137, 655 (1936).

\title{
Erratum: Heat Conductivity of Tungsten and the Cooling Effects of Leads upon Filaments at Low Temperatures
}

\author{
Irving Langmuir and John Bradshaw Taylor, General Electric Company
}

(Phys. Rev. 50, 68 (1936))

I $N$ the last sentence of the abstract the equation was misprinted. The correct equation is:

$$
\log \lambda=0.9518-0.30 \log T \text {. }
$$

